\title{
TIMETOAST TIMELINE COMO PROPOSTA PARA LINHA DO TEMPO INTERATIVA E USO EM EDUCAÇÃO MATEMÁTICA
}

\section{TIMETOAST TIMELINE AS A PROPOSAL FOR THE INTERACTIVE TIMELINE AND USE IN MATHEMATICAL EDUCATION}

\author{
Anna Beatriz de Andrade Gomes ${ }^{1}$; Giselle Costa de Sousa ${ }^{2}$
}

\begin{abstract}
RESUMO
O Timetoast Timeline é um site que permite fazer linhas do tempo de maneira interativa e de melhor visualização, por meio de uma narrativa cronológica com informações como período do acontecimento e/ou pessoa, imagens e links vinculados e ainda com informações descritivas adicionais. O objetivo geral deste artigo é apresentar as funções, ferramentas e possibilidades que o Timetoast Timeline contém, visando as possibilidades de produção de trabalhos voltados para a História da Matemática (HM) na Educação Matemática. Por meio de um estudo de abordagem qualitativa feito mediante recursos como tutoriais e exploração do próprio site, apresentamos como utilizá-lo para a criação de linhas do tempo e gerenciamento de informações da HM. Além disso, exibiremos como utilizamos essa plataforma para produzir uma linha do tempo interativa selecionando tópicos de HM com ênfase em Geometria, baseando-se em trabalhos publicados anteriormente, buscando fomentar atividades-históricas-com-tecnologia. No Timetoast Timeline, adicionamos em uma ordem cronológica todos os personagens encontrados no Mactutor History of Mathematics Archive, site que apresenta uma linha do tempo e biografia de estudiosos do campo da matemática, que trabalharam com estudos de natureza geométrica, seja na própria matemática ou em outras áreas como astronomia, engenharia e entre outros, e, adjunto, período em que viveu, um tópico histórico sobre o mesmo, uma imagem que o representa, algumas de suas obras relacionadas a sua produção e onde tais documentos estão disponibilizados (sejam nos formatos digitais ou físicos, quando encontrados). Como resultado, além da apresentação desse recurso, produzimos uma linha do tempo parcial, baseando-se no levantamento obtido durante a pesquisa cujo processo de produção revelamos neste trabalho.
\end{abstract}

Palavras-chave: Timetoast Timeline; História da Matemática; Educação Matemática; Geometria; atividades-históricas-com-tecnologia.

\footnotetext{
ABSTRACT

The Timetoast Timeline is a website that allows you to make timelines in an interactive and better visualization way, through a chronological narrative with information such as the period of the event and / or person, images and linked links and with additional descriptive information. The general objective of this article is to present the functions, tools and possibilities that Timetoast

${ }^{1}$ Graduação em andamento pela Universidade Federal do Rio Grande do Norte (UFRN). Natal, RN, Brasil. Endereço para correspondência: Terceira Travessa da Floresta,09, Nossa Senhora da Apresentação, Natal, RN, Brasil, CEP: 59114-003. E-mail: gomesbeatriz.anna@gmail.com

ORCID iD: https://orcid.org/0000-0002-7642-4526

${ }^{2}$ Doutora. Universidade Federal do Rio Grande do Norte (UFRN). Professora associada do DMAT (UFRN), Natal, RN, Brasil. Endereço para correspondência: Cirilo Moreira, 261 - Nazaré, Natal, RN, Brasil, CEP: 59062-130. E-mail: gisellematufrn@ gmail.com

(iD) ORCID iD: https://orcid.org/0000-0003-0213-4179
} 
Timeline contains, the possibilities of producing works focused on the History of Mathematics (HM) in Mathematics Education. Through a study of a qualitative approach made using resources such as tutorials and exploration of the site itself, we present how to use it for the creation of timelines and management of HM information. In addition, we will show how we use this platform to produce a selected interactive HM timeline with an emphasis on Geometry, based on previously published works, seeking to foster historical-with-technology activities. In the Timetoast Timeline, we added in a chronological order all the characters found in the Mactutor History of Mathematics Archive, a website that presents a timeline and biography of scholars in the field of mathematics, who worked with studies of a geometric nature, either in mathematics itself or in other areas such as astronomy, engineering and others, and, adjunct, period in which he lived, a historical topic about him, an image that represents him, some of his works related to his production and where such documents are made available (whether in the formats digital or physical, when found). As a result, in addition to the presentation of this feature, we produced a partial timeline, based on the research which the production process was revealed in this work.

Keywords: Timetoast Timeline; History of Mathematics; Mathematics Education; Geometry; historical-activities-with-technology. 


\section{Introdução}

O site Timetoast Timeline ${ }^{3}$ apresenta uma plataforma em inglês que permite o usuário produzir linhas do tempo sobre qualquer assunto e qualquer período desejado, mostrando, assim, uma narrativa de maneira cronológica e compartilhada. Por meio dele, é possível contar narrativas de maneira cronológicas e visualizá-las de forma organizada e comunicativa, relacionando épocas, dados descritivos, imagens e links.

O presente artigo apresenta as possibilidades desta plataforma para a produção de trabalhos, principalmente na área de História da Matemática, por meio de linhas do tempo interativa para contar narrativas de personagens históricos matemáticos, cronologias de estudos feitos em determinadas áreas da matemática, e entre outras possibilidades.

Além disso, apresentamos nossa experiência quanto ao uso da plataforma para produzir uma linha do tempo interativa em Geometria com o objetivo de fomentar atividades-históricas-com-tecnologia por meio de tópicos de História da Matemática. Trata-se de uma pesquisa em andamento, portanto, expomos apenas os dados tratados até agora.

A pesquisa citada é um desdobramento de pesquisas anteriores que estudam a possibilidade da aliança entre História da Matemática (HM) e Tecnologias Digitais de Informação e Comunicação (TDIC) para o uso no Ensino da Matemática. E esta aliança se concretiza por meio de atividades-históricas-com-tecnologias, que são atividades que abordam problemas históricos investigativos aliados a tecnologias.

Considerando os objetivos citados anteriormente, passamos para os caminhos seguidos nesta pesquisa.

\section{Percurso Metodológico}

A metodologia utilizada no trabalho foi de abordagem qualitativa. A pesquisa qualitativa busca abranger os aspectos que representam qualidade da pesquisa e suas subjetividades, sem focar no lado quantitativo e seus processos, como apresenta Neves (1996):

Dela faz parte a obtenção de dados descritivos mediante contato direto e interativo pesquisador com a situação objeto de estudo. Nas pesquisas qualitativas, é frequente que o pesquisador procure entender os fenômenos, segundo a perspectiva dos participantes da situação

\footnotetext{
${ }^{3}$ Link de Acesso: https://bit.ly/2M6mb9G
} 
estudada e, a partir, daí situe sua interpretação dos fenômenos estudados.(NEVES, 1996, p. 01)

Ou seja, por meio do nosso objeto de estudo, fizemos o levantamento de informações descritivas, procurando analisar, compreender e, assim, por meio do tratamento os dados coletados, trazer informações proficientes e úteis neste trabalho.

Utilizando o método qualitativo, estudamos o funcionamento do site Timetoast Timeline, que é o nosso objeto de pesquisa neste trabalho e incluimos uma apreciação de suas ferramentas, funcionalidade, possibilidades e restrições, de maneira a ser utilizado para o uso na História da Matemática (particularmente a organização de levantamentos realizados), contribuindo para produção de trabalhos na área da Educação Matemática.

A plataforma é, de certa forma, intuitiva e fácil de usar, porém, para nos apropriarmos melhor dela, procuramos instruções ou exemplos do próprio site e/ou tutoriais que pudessem auxiliar. A maioria dos tutoriais foram encontrados em inglês pelo YouTube, alguns deles foram: TimeToast Student Tutorial (2017), TimeToast Timeline Tutorial (2017), Using Timetoast Timeline Maker (2021), Timetoast - Criação de linhas do tempo (2016), entre outros.

Com isso, exibiremos a seguir todas as informações coletadas sobre a plataforma TimeToast Timeline e como o utilizamos para produzir uma linha do tempo com tópicos de História da Matemática voltados para Geometria.

\section{Timetoast Timeline}

Para começar a utilizar o site, é necessário criar uma conta ou já ter uma conta no mesmo e escolher um tipo de plano. Há 3 tipos de planos: Public (Público), Basic (Básico) e Pro (Abrviação de Profissional). A diferença entre os planos é a presença de alguns recursos. No plano público, é possível publicar linhas ilimitadas, uma única pessoa (a que criou) pode editá-la e há alguns anúncios durante o uso, porém, nada que atrapalhe a utilidade do site. Já nos outros dois, há a possibilidade de mais de um usuário editar, mudar as cores da linha, fazer comentários e criar grupos. É possível mudar de plano após selecionar um deles.

Ao criar a conta e selecionar um dos planos, já é possível iniciar a própria linha. Na página inicial do Timetoast, há alguns exemplos de linhas feitas por outros usuários. A partir delas, dá para ter uma ideia inicial de como funciona a linha depois de finalizada. 
O primeiro passo ao selecionar a opção Add Timeline + (Adicionar Linha do Tempo), na aba de Linhas do Tempo, será responder um formulário (Figura 1), que permite escolher o nome da linha criada, se ela iniciará como rascunho ou pública, selecionar uma categoria (ou sem categoria) e adicionar uma imagem que represente a linha.

Figura 1 - Formulário para criação de linha do tempo

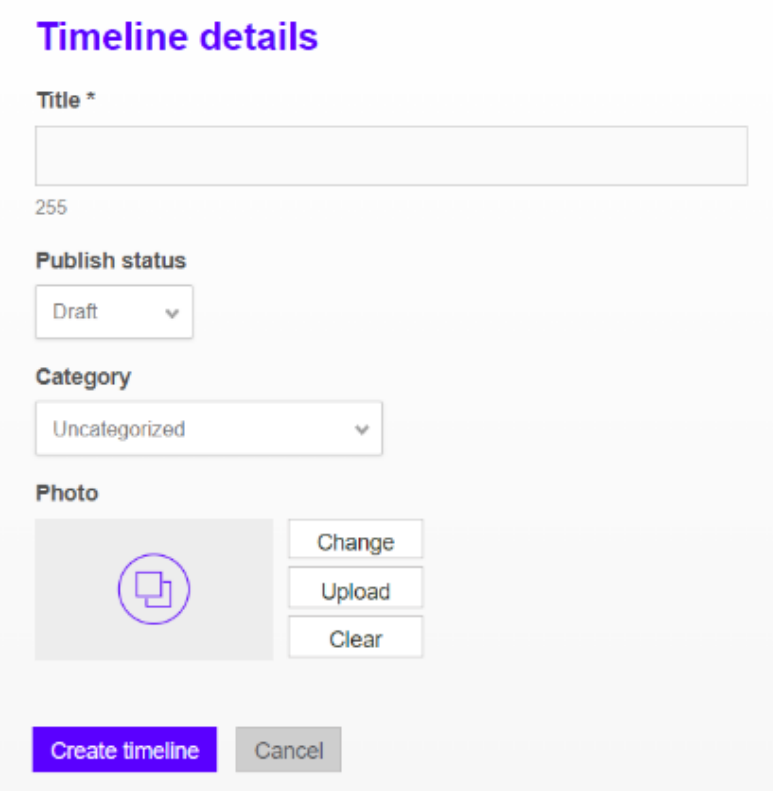

Fonte: Site Timetoast Timeline (2021)

O espaço de Title é onde vai ser adicionado o título da linha do tempo. Já em Publish status (Status de Publicação) há duas opções: Draft (Rascunho) ou Public (Público), ou seja, é nessa parte onde é decidido se a sua linha do tempo está pronta para ser publicada ou se ficará como rascunho. Logo após, seleciona-se a Category (Categoria) da linha. Nesta parte, é importante selecionar uma categoria, pois, essa é uma das formas de sua linha do tempo ser encontrada mais facilmente no site.

Para a seleção, há as seguintes categorias: Music (Música), Film (Filme), Science and Technology (Ciência e Tecnologia), Business (Negócios), Politics (Política), Biography (Biografia), Art and Culture (Arte e Cultura), Personal (Pessoal) e History (História). Além disso, caso a sua linha não se encaixe em algumas das categorias, pode ser selecionada a opção Uncategorized (Sem categoria). Por fim, há a possibilidade de selecionar uma imagem que represente a linha, porém, essa opção não é obrigatória para a criação da mesma. 
Após criada, a linha do tempo já pode ser alimentada com suas prórpias informações. Há duas maneiras de colocar essas informações na linha. A primeira é como evento. Para isso, deverá ser selecionada a opção Add event (Adicionar evento), preencher o formulário (Figura 2) e apertar em Create event (Criar Evento).

Figura 2 - Formulário para detalhes do Evento

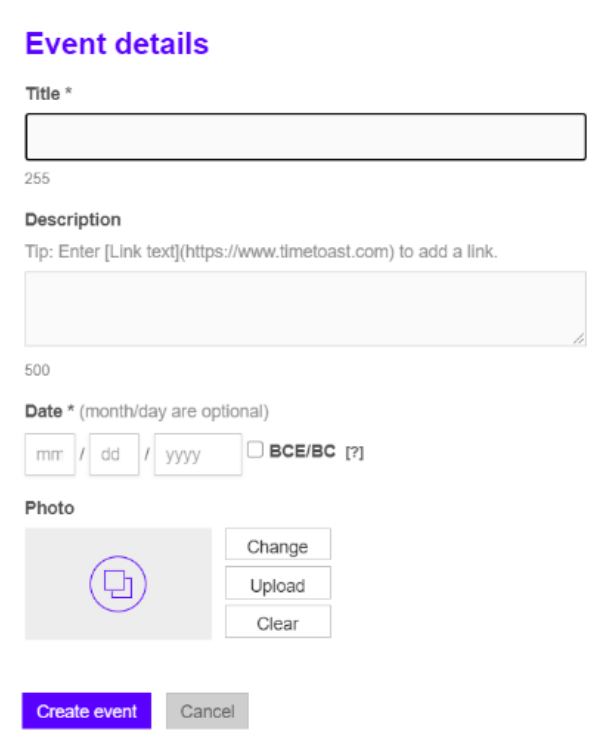

Fonte: Site Timetoast Timeline (2021)

Na opção de Title, deverá ser colocado o título do evento. Em Description (Descrição), é possível preencher com informações ou links relacionados ao evento que está para ser adicionado. Na área de Date (Data), por se tratar de um site em inglês, a data deverá ser colocada na seguinte ordem: mounth/day/year (Mês/Dia/Ano), porém, só é obrigatório colocar o ano do evento. Além disso, caso o evento tenha acontecido Antes da Era Comum, é necessário selecionar a opção BCE/BC: Before Comum Era/Before Christ (Antes da Era Comum/Antes de Cristo). Também pode ser adicionada uma imagem referente ao evento.

A segunda opção de apresentar informações na linha do tempo é a de Espaço de Tempo. Para isso, será selecionada a opção Add timespan + (Adicionar espaço de tempo) e preencher com as informações, como apresenta a Figura 3. 
Figura 3 - Formulário para detalhes do Espaço de Tempo

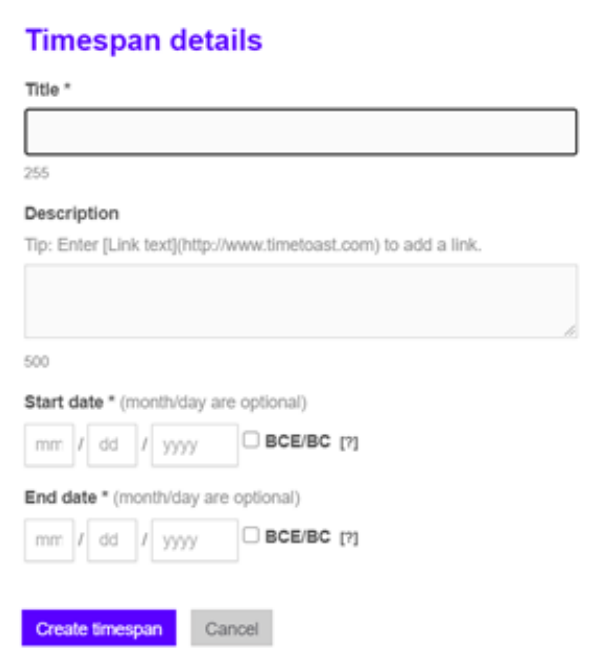

Fonte: Site Timetoast Timeline (2021)

Da mesma maneira que o evento, o formulário espaço de tempo inicia com o título e descrição. Na área que é destinada para preencher as informações sobre a data, ele apresenta o Star date (Data inicial) e End date (Data final), ou seja, é possível adicionar a data em que começa e em que termina o espaço de tempo. Esta é uma das restrições do evento, que não permite adicionar data de finalização. Já no espaço de tempo, a restrição está na falta da possibilidade de adicionar qualquer tipo de imagem.

Logo, após criados os eventos, eles já estarão disponíveis para serem visualizados e compartilhados na sua linha do tempo. No botão de menu, Para exemplificar como ficará uma possível criação de linha, trouxemos um exemplo de visualização (Figura 4) e dois exemplos (Figura 5 e 6 ) de como seria exposto um tópico sobre o personagem matemático histórico Paul Guldin (1577-1643). 
Figura 4 - Exemplo de Linha do tempo

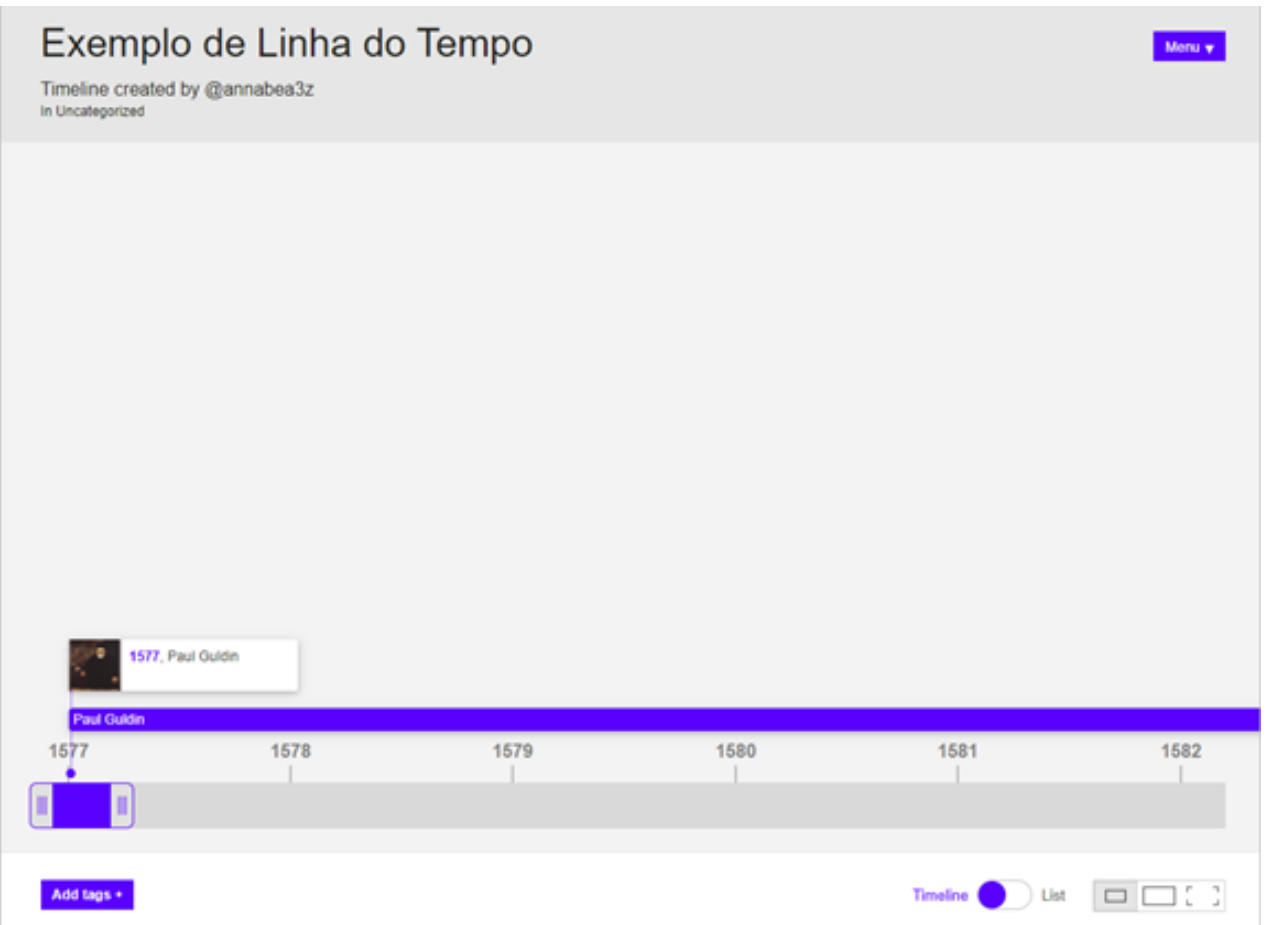

Fonte: Site Timetoast Timeline (2021)

$\mathrm{Na}$ figura 4 é possível visualizar o nome da linha do tempo, exemplo de linha do tempo, por qual usuário foi criada e sua categoria. No canto superior direito, onde tem a opção Menu, é possível selecionar para editar a linha do tempo, caso seja o usuário responsável pela linha, na opção Edit Timeline. Além disso, Print, para imprimir a linha e Share, para gerar um link onde é possível compartilhá-la.

No canto inferior esquerdo, em Add tags + (Adicionar tags), há a possibilidade de adicionar tags ou palavras chaves para que, quando algum outro usuário pesquise por elas, seja possível encontrar sua linha do tempo ou semelhantes a ela. Já no canto infernior direito, há a opção de selecionar se quer a linha do tempo em forma de Timeline (Linha), como apresentado no exemplo, ou em forma de Lista (Lista), onde todos os eventos e espaços de tempo são listados em ordem crescente. Mais ainda, há 3 formas de apresentar a linha de acordo com o tamanho que deseja.

Na linha propriamente dita, há um evento, o retângulo com a imagem, nome e ano em que nasceu do Paul Guldin. Ela fica fixa apenas na data inicial, como dito anteiormente neste trabalho. O espaço de tempo é exposto em forma de linha, que inicia 
na primeira data apresentada e termina na segunda data. É possível clicar nas duas opções para abrir uma aba com as informações adicionadas ao editar a linha (Figura 5 e 6 )

Figura 5 - Exemplo de evento

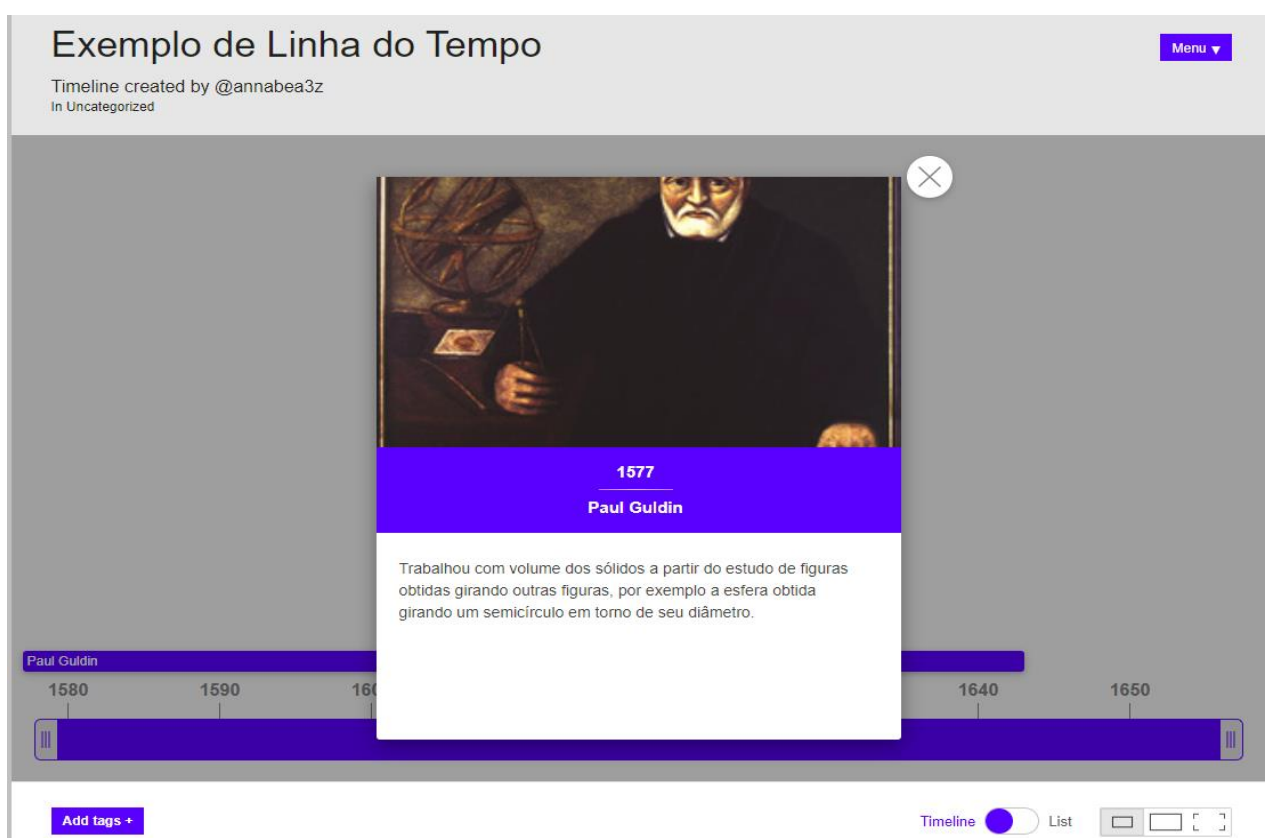

Fonte: Site Timetoast Timeline (2021)

Figura 6 - Exemplo de espaço de tempo

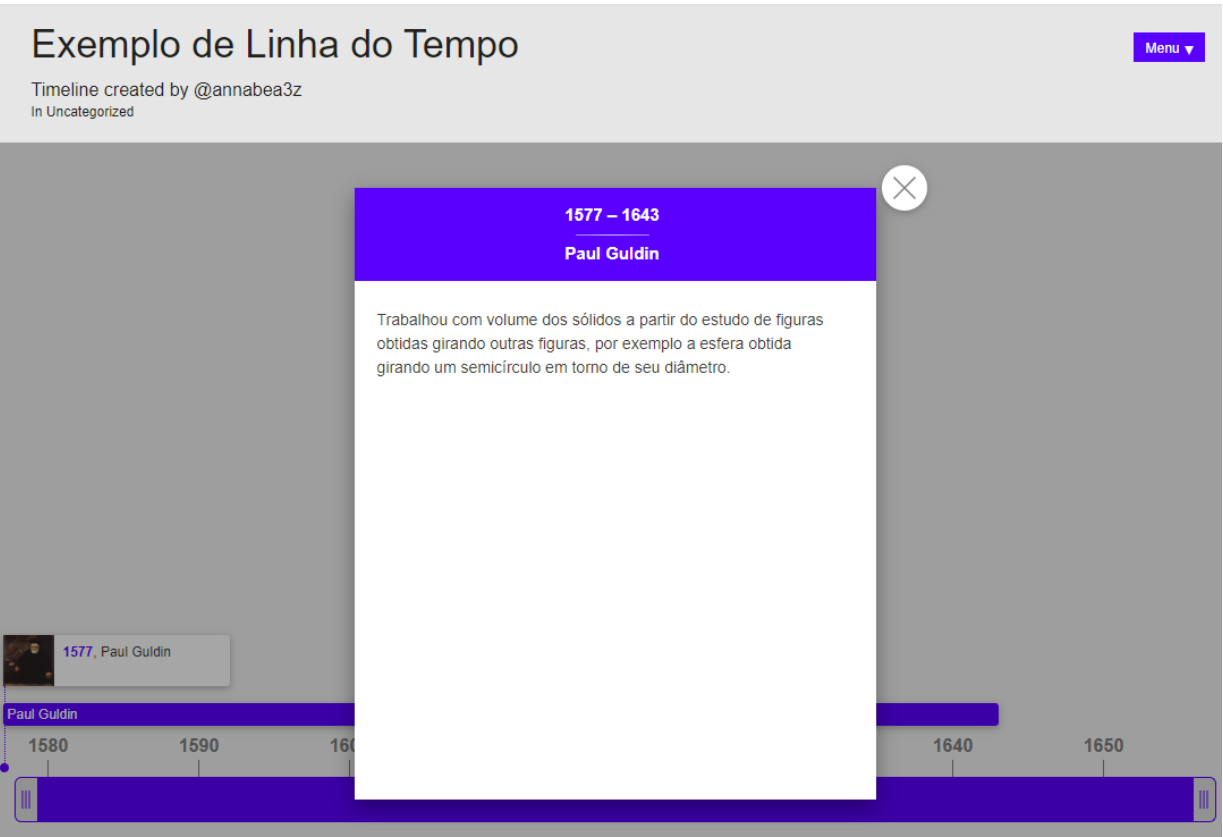

Fonte: Site Timetoast Timeline (2021) 
Ao clicar no evento ou no espaço de tempo, abrem as abas apresentadas na Figura 5 e 6, respectivamente. Nota-se as restrições citadas anteriormente como não poder adicionar a data final de um evento e imagem no espaço de tempo, porém, mesmo com essas restrições, o site apresenta uma ótima plataforma para trabalhar com Linhas do Tempo de História, principalmente na área de História da Matemática, pois permite que o usuário visualize ainda melhor a ordem cronológica dos acontecimentos, fazendo com que haja uma melhor organização de dados. Logo, abre possibilidades para alunos da educação básica, do ensino superior e professores façam uso desta ferramenta em sala de aula para melhor acesso a informações, também abre possibilidades para trabalhar essa e outras formas de cronologias da linha em sala de aula.

Após toda a análise apresentada sobre o site Timetoast Timeline, mostraremos a seguir como a ferramenta está sendo utilizada em um projeto de pesquisa de iniciação científica em andamento na área da aliança entre História da Matemática e Tecnologias Digitais de Informação e Comunicação (TDIC) como ferramenta para a educação matemática.

\section{Linha do tempo interativa Geométrica}

A pesquisa apresentada nesta seção é vinculada ao projeto Conexões Potenciais entre História da Matemática e Tecnologias Digitais de informação e Comunicação: Aporte para fomento de atividades-históricas-com-tecnologias (PROPESQ/UFRN), que estuda a possibilidade da aliança entre História da Matemática e Tecnologia por meio da Investigação Matemática como proposta para o Ensino da Matemática.

Nas pesquisas anteriores vinculadas ao este projeto, Costa e Sousa (2017) realizaram um levantamento de trabalhos onde apresentavam a aliança entre História da Matemática e Tecnologias Digitais de Informação e Comunicação. Após esse levantamento, notaram que há poucos trabalhos voltados para esta direção e estabeleceram parâmetros (Metodologia, Tema, Software e Cunho Educacional), baseando-se na análise dos trabalhos obtidos, que apresentavam com maior frequência. O parâmetro Tema mais frequente nestes trabalhos foi a Geometria.

Em seguida, Gomes e Sousa (2020) definiram o que são atividades-históricascom-tecnologia como sendo atividades que trabalham com problemas históricos com auxílio de tecnologias por meio da investigação e, para mais, definiram também 
elementos estruturantes destas atividades, como uma forma de dar aporte para a produção de atividades nesta direção.

Como uma maneira de fomentar atividades-históricas-com-tecnologia, a pesquisa realizada agora tem o objetivo de fazer uma seleção de tópicos de História da Matemática voltados para Geometria que podem ser tratados na aliança como tema histórico das referidas atividades para a sala de aula, seja na educação básica ou no ensino superior, e organizar tal levantamento numa linha do tempo ${ }^{4}$, baseando-se nos resultados dos trabalhos anteriores apresentados o projeto maior apresentado anteriormente. Para isto, utilizamos o site MacTutor History of Mathematics Archive ${ }^{5}$ (O'CONNOR; ROBERTSON, 2019) e realizamos uma pesquisa, que está em andamento, de personagens históricos que produziram resultados de caráter geométrico ao longo da vida, ou seja, que fez algum tipo de contribuição ou estudos na área da geometria.

Para o levantamento de dados, optamos por, incialmente, produzir planilhas dividindo-as por períodos. Até o momento, há três planilhas: da Idade Antiga, Idade Média e Idade Moderna, baseando-se na divisão de períodos feita por Vázquez, Rey e Boubée (2008). Nestas planilhas, separamos cinco colunas, referentes aos estudiosos trabalhados, nomeadas de Período, Nome, Contribuições, Obras e Disponibilidade das Obras, como apresentado na Figura 7.

\footnotetext{
${ }^{4}$ Salientamos que esta é uma possibilidade de organização dos dados, mas que não estamos necessariamente considerando a matemática e sua história como linear e progressista.

${ }^{5}$ Link de Acesso: https://bit.ly/3qwRQzT
} 
Figura 7 - Recorte da Planilha com Estudiosos que trabalharam com Geometria

\begin{tabular}{|c|c|c|c|c|}
\hline PERIOODO & NOME & CONTRIBUIÇÕES & OBRAS & DISPONIVEL \\
\hline $\begin{array}{c}\text { Aproximadamente } 800 \\
\text { A.E.C. }\end{array}$ & Baudhayan & $\begin{array}{l}\text { Em sua obra, utilizava valores de } \\
\pi \text { com certo grau de precisão } \\
\text { para diferentres contruções } \\
\text { circulares e, também, apresentou } \\
\text { algo próximo do que conhecemos } \\
\text { como teorema de pitágoras. }\end{array}$ & $\begin{array}{c}\text { Baudhayan's Sulbasutra (data } \\
\text { desconhecida) }\end{array}$ & Obra perdida. \\
\hline 750 A.E.C. - 690 A.E.C. & Manava & $\begin{array}{l}\text { Sua obra continha construções } \\
\text { aproximadas de círculos de } \\
\text { retângulos e quadrados de } \\
\text { círculos, que apresentavam o } \\
\text { valor aproximado para } \pi .\end{array}$ & $\begin{array}{c}\text { Manava's Sulbasutra (data } \\
\text { desconhecida) }\end{array}$ & Obra perdida. \\
\hline 624 A.E.C. - 547 A.E.C. & Tales de Mileto & $\begin{array}{c}\text { Foi atribuido a Tales a descoberta } \\
\text { do Teorema de Tales quando o } \\
\text { matemático teve a ideia de } \\
\text { calcular a altura de uma piramide } \\
\text { por meio de sua sombra. Além } \\
\text { disso, descobriu que os ângulos } \\
\text { da base de um triângulos isóceles } \\
\text { são iguais, que um diâmetro } \\
\text { divide um círculo em partes iguais } \\
\text { e entre outros. }\end{array}$ & $\begin{array}{c}\text { Não teve obras de geometria } \\
\text { publicadas. }\end{array}$ & - \\
\hline 569 A.E.C. - 475 A.E.C. & Pitágoras de Samos & $\begin{array}{l}\text { Atribui-se a ele o Teorema de } \\
\text { Pitágoras que se refere a um } \\
\text { triângulo retângulo em que o } \\
\text { quadrado da hipotenusa é igual à } \\
\text { soma dos quadrados dos catetos. } \\
\text { Além disso, notou que a soma } \\
\text { dos ângulos de um triângulo é } \\
\text { igual a dois ângulos retos e } \\
\text { pesquisou sobre os cincos sólidos } \\
\text { regulares e álgebra geométrica. }\end{array}$ & $\begin{array}{c}\text { Não teve obras de geometria } \\
\text { publicadas. }\end{array}$ & - \\
\hline
\end{tabular}

Fonte: Elaborado pelas autoras (2020)

Por meio destas planilhas, organizamos os dados coletados da seleção dos tópicos e isso facilitou transferir as informações para o site de modo a alimentar a linha do tempo interativa do TimeToast. Havia outras alternativas ao Timetoast Timeline, porém, após a busca e estudo de outras plataformas, notamos que a mesma apresentava uma plataforma mais completa e que suportava todos os dados que planejamos acrescentar.

Intitulamos a linha do tempo interativa de Matemáticos em Geometria e a categorizamos na área da História. Como tratamento dos dados, utilizamos a opção espaço de tempo para separar os períodos (Idade Antiga, Média e Moderna, por enquanto), e a opção de eventos para definir os estudiosos matemáticos (Figura 8). 
Figura 8 - Linha do Tempo: Matemáticos em Geometria

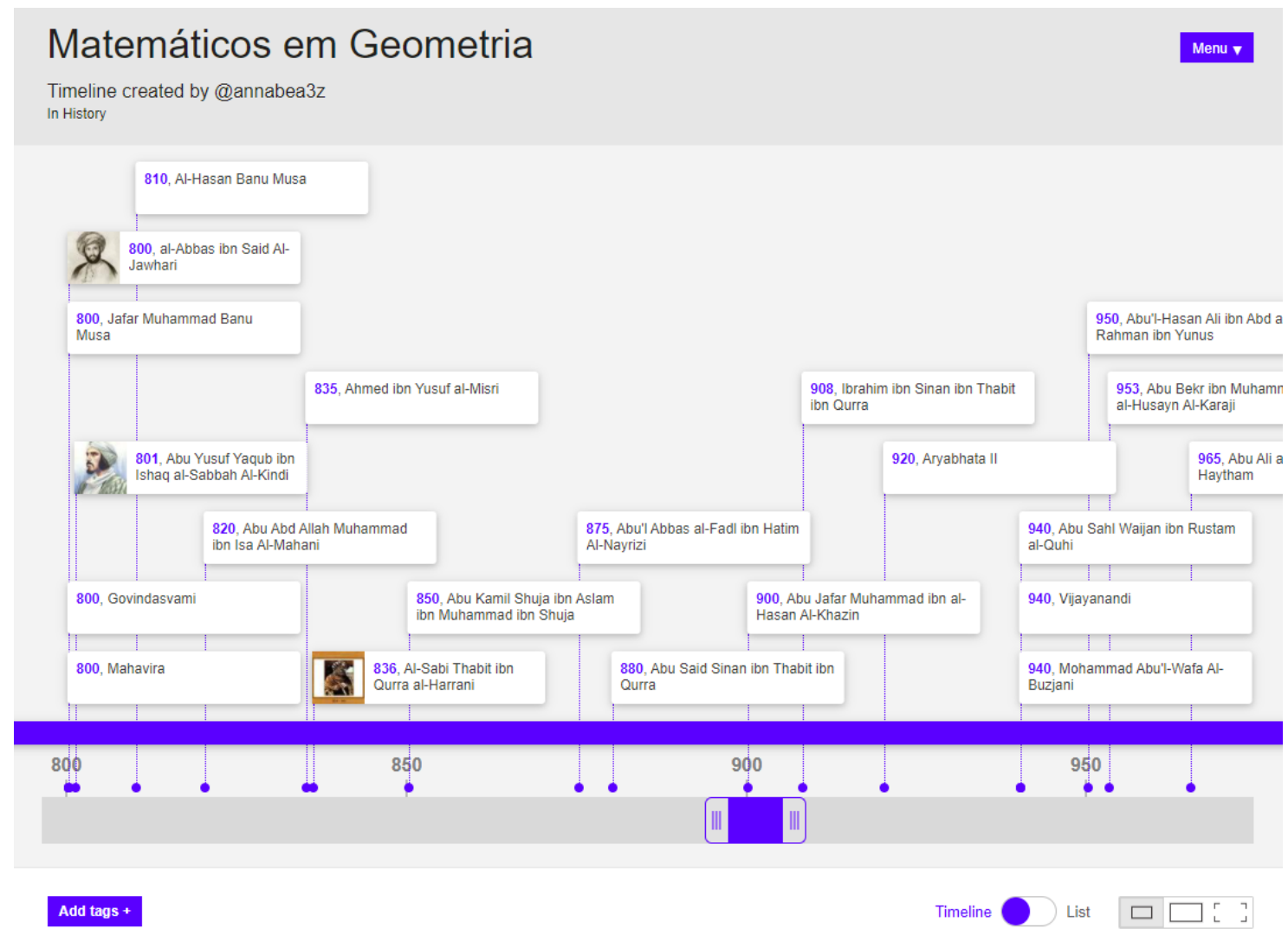

Fonte: Site Timetoast Timeline

O período representado na Figura 8 corresponde aos anos antes e depois de 900 Antes da Era Comum. Como é uma linha do tempo extensa, há um botão abaixo da mesma onde é possível arrastá-lo de acordo com o período que deseja ser visualizado. Ao acrescentarmos a descrição do evento, que seria o tópico histórico, a obra e o link para obra, notamos que não dá para fazer um espaço entre os 3 , então, juntamos todos os três em um único texto.

Optamos por fazer o uso de imagens para alguns matemáticos selecionando as pinturas/desenhos/caricaturas que apareciam predominantemente nas pesquisas relacionadas aos sábios estudados, porém, as que apareciam a mesma imagem para mais um, escolhemos não incluir. Elas foram postas como uma forma de comunicação e informação para quem estará fazendo uso da linha do tempo. Contudo, pretendemos incluir a informação de que, em muitos casos, não correspondem a fotos dos personagens, pois inclusive, a depender do período, esse recurso não existia. Também salientaremos as fontes. 
Ao finalizarmos as planilhas catalogando todos os matemáticos do MacTutor que consideramos trabalhar com Geometria, concluiremos uma primeira versão da linha do tempo interativa do Timetoast. Contudo, tal levantamento seguirá para outras fontes que permitam, inclusive, incluir personagens não mencionados pelo MacTutor e/ou que tinham trabalhos em outras áreas (que não necessariamente matemáticas, mas que envolvem Geometria) de modo a fomentar novas versões da linha e/ou uma alimentação desta periodicamente. Por fim, trazemos as considerações finais desse artigo.

\section{Considerações finais}

Este trabalho teve por objetivo apresentar e familiarizar a plataforma TimeToast Timeline como uma proposta para a criação de linhas do tempo digitais e interativas com uso na Educação Matemática, particularmente, em HM. Ela contém algumas ferramentas que permitem trazer informações de maneira mais atrativa e, por este motivo, notamos as possibilidades de usá-la para trabalhos, principalmente voltados para a área de História da Matemática, seja para contar a vida de um estudioso matemático, o desenvolvimento de uma área/conceito durante o tempo, entre outras possibilidades. Assim, o seu uso pode ser útil para o Ensino da Matemática, como uma plataforma de acesso a cronologias históricas ao utilizar a tendência História da Matemática em sala de aula.

Para exemplificar, utilizamos uma pesquisa realizada pelas autoras, que está em andamento, afim de exemplificar o uso do Timetoast na História da Matemática (HM), especialmente, na organização de dados pesquisados. Por meio de um levantamento feito no site Mactutor, selecionamos personagens históricos que trabalharam e fizeram contribuições na Geometria com o propósito de fomentar atividades-históricas-comtecnologia. E, com esta pesquisa, esperamos fomentar atividades-históricas-comtecnologia, que influenciarão diretamente na maneira de ensinar matemática utilizando a história.

Além disso, visando outras propostas futuras para o ensino da matemática por meio da História da Matemática, o Timetoast Timeline também pode ser utilizando para trabalhar outras vertentes da HM, como linhas do tempo exclusivas de matemáticos brasileiros, mulheres na matemática, matemáticos que trabalharam com as outras diversas áreas, e entre outros. 


\section{Referências}

BENNETT, Rob. TimeToast Timeline Tutorial. Youtube. 2020. Disponível em: https://www.youtube.com/watch?v=McpYUX9ozvU\&t=406s . Acesso em: $24 \mathrm{de} \mathrm{fev.}$ 2021. 10:51

BOYLE, Daniel. Using Timetoast Timeline Maker. Youtube. Disponível em: https://www.youtube.com/watch?v=I7M4MiGFa5w\&t=1s . Acesso em: 24 de Fev. $202105: 31$

COSTA, Allyson Emanuel Januário da; SOUSA, Giselle Costa de. Investigando a Conjunção entre História da Matemática e Tecnologias de Informação e Comunicação, por meio de um Levantamento Bibliográfico em Eventos Internacionais de Educação Matemática. Revista Boletim Cearense de Educação e História da Matemática, Ceará, v.04, n.11, 06 - 21. (2017).

NEVES, José Luis. Pesquisa Qualitativa - Características, Usos e Possibilidades. Caderno de Pesquisas em Administração, São Paulo, v.1, nº 3, 2 SEM./1996.

O'CONNOR, John J.; ROBERTSON, Edmundo F.. Mactutor History of Mathematics archive. Disponível em: https://www-history.mcs.st-and.ac.uk/. Acesso em: 02 nov. 2019.

PAYSEN, Erin. Timetoast Student Tutorial. Youtube. 2017. Disponível em: https://www.youtube.com/watch?v=FLzvlzXQLco\&t=1s. Acesso em: 24 de fev. 2021. 06:05

SOUSA, Giselle Costa De; GOMES, Anna Beatriz De Andrade. Aporte para a promoção de atividades-históricas-com-tecnologia. Research, Society and Development, v. 9, n. 5, p. 1689-1699, 2020.

UBN - CEAD. Timetoast - Criação de linhas do tempo. Youtube. 2020. Disponível em: https://www.youtube.com/watch?v=a8QfLGqafQA . Acesso em: 247 de fev. 2021. $6: 46$

VÁZQUEZ, P. S.; REY, G.; BOUBÉE, C.. "El concepto de función a través de la Historia". Revista Ibero americana de Educação Matemática. v. 4, n. 6, pp. 141151, Dez. 2008.

Recebido em: 02 / 03 / 2021

Aprovado em: 26 / 04 / 2021 